\title{
IS INFLUENZA VACCINE ASSOCIATED WITH COVID-19 INFECTION SEVERITY: META-ANALYSIS
}

\author{
Shinta Nur Atikah \\ Masters Program in Public Health, Universitas Sebelas Maret
}

\begin{abstract}
Background: Recently, several studies have suggested a possible protective effect of the influenza vaccine against SARS-CoV-2. Although no cross-reactivity between influenza-induced antibodies and SARS-CoV-2 protection has been demonstrated, several theorized mechanisms of the potential protective effect of influenza vaccination have been proposed in the recent studies. Alternatively, influenza vaccination's potential protective effect may be explained by its ability to stimulate the activation of natural killer cells, the levels of which have been found to be considerably decreased in moderate and severe SARS-CoV-2 cases. This study aimed to investigate the effect of influenza vaccine on COVID-19 infection severity.

Subjects and Method: A systematic literature search was conducted in multiple databases including Pubmed, Google Scholar, and Sciene Direct, to identify randomized control trial published from 2020 to 2021. The following search terms were used: "COVID-19" OR "COVID-19 Severity" AND "Vaccine Influenza". The inclusion criteria were English full text and randomized controlled trial. The articles were filtered using PICO model, including: (1) Population= COVD-19 patients, (2) intervention= influenza vaccine, (3) comparison $=$ without influenza vaccine, and (4) outcome $=$ severity of COVID-19 infection. The inclusion criteria were English full-text, observational study, and reported adjusted Odds Ratio (aOR). The systematic review was carried out according to the PRISMA diagram. Data analysis were performed using RevMan 5.3.

Results: A meta analysis involved 7 studies from Italia, United States, Spain, and Taiwan showed that influenza vaccine reduced severity of COVID-19 infection (aOR= $0.73 ; 95 \% \mathrm{CI}=0.58$ to $0.92 ; \mathrm{p}=0.05$ )

Conclusion: Influenza vaccine reduces severity of COVID-19 infection.
\end{abstract}

Keywords: influenza vaccine, severity, COVID-19 infection

Correspondence:

Shinta Nur Atikah. Masters Program in Public Health, Universitas Sebelas Maret. Jl. Ir. Sutami 36A, Surakarta 57126, Central Java. Email: shintaatikah@student.uns.ac.id. Mobile: 081295254862 